\title{
SHEDDING LIGHT ON SOUND: THE FUSION OF ACOUSTO-OPTIC AND B-MODE ULTRASOUND IMAGING
}

\author{
Ronald A. Roy, Lei Sui, Charles A. DiMarzio*, and Todd W. Murray \\ Aerospace and Mechanical Engineering, Boston University, Boston, MA 02215 \\ *Electrical \& Computer Engineering, Northeastern University, Boston, MA 02115
}

\begin{abstract}
Ultrasound modulation of light can be used to image optical properties in highly diffusive media such as biological tissues. Much of the previous work in this area employed continuous wave (cw) ultrasound to pump the acousto-optic response. We argue in favor of using pulsed ultrasound, based on regulatory considerations related to bioeffects and enhanced axial resolution along a scan line. We describe a system in which a commercial ultrasound scanner was combined with a photorefractive crystal (PRC) based optical detection scheme to generate simultaneous 3D images of acoustic and optical properties of optically absorbing inclusions embedded in excised biological tissue. Representative images are presented that demonstrate the utility of this dual mode imaging technique. Technological limitations are discussed, as are plans for future work.
\end{abstract}

\section{INTRODUCTION}

The subsurface imaging of tissues is a rapidly expanding field of study. Traditional techniques based on x-ray, MR, and ultrasound (US) contrast are well established and each offers its own unique advantages and disadvantages. For example, xrays are very penetrating and have proven effective at imaging bone structure, whereas US provides structural and blood flow information based (primarily) on contrast in compressibility. There are a host of other potentially useful modalities, including MR and ultrasound elastography [1,2] (delineates tissue elastic modulus), shear wave imaging [3] (delineates tissue shear modulus), and electrical impedance tomography [4] (maps spatially-dependent electrical properties), to name but a few. The choice of imaging modality depends on a number of factors, including temporal/spatial resolution and the physical nature of the contrast that is deemed relevant to the condition one seeks to image or sense. Indeed, there may be instances where more than one imaging modality is required to resolve ambiguities and enhance the clinician's ability to diagnose. For example, one might combine MR with ultrasound to both image the boundaries of a tumor (MR) and quantify tumor perfusion (contrast enhanced US). Problems associated with physical access and image registration complicate matters, particularly when attempting to obtain multi-mode images of tissues that are evolving with time.
One of the more sensitive techniques for delineating tissue structures and types involves optical scattering and absorption [5]. For example, the absorption of light by hemoglobin serves as a sensitive indicator of blood volume and vascular structure. Frequency-dependent optical attenuation can yield spatially and temporally dependent functional information. The problem is, when light propagates through tissue it is highly scattered. Images based on the resulting diffuse optical field have poor spatial resolution. In the early 90's researchers began combining US with diffuse light in an attempt to enhance spatial resolution in a technique referred to as acousto-optic imaging (AOI). When photons enter the sound beam, they interact with scattering sites that undergo periodic translational motions under acoustic forcing [6]. This, along with pressureinduced changes in the index of refraction, results in a photon frequency shift that is modulated at the same frequency as the US. Thus one can selectively sense only those photons that emanate from volume of tissue exposed to ultrasound. In a sense, these photons emanate from a "virtual light source" (VLS) whose dimensions are limited to the sound beam.

There are a number of ways that this virtual source can be put to use to generate images. Probably the simplest approach is to spatially translate the US beam and monitor the changes in the VLS photon flux. As one traverses regions possessing differing optical absorption, the effective strength of the VLS changes, leading to the contrast observed in AO images. The resolution in AOI is determined by the US beam, thereby allowing for an effective measure of subsurface optical properties with ultrasound resolution. This technique has been successfully employed by a number of investigators using both pulsed and cw ultrasound to pump the acousto-optic (AO) signal [7]. These will not be reviewed here, except to point out that much of the previous work using pulsed US was plagued by poor signal-to-noise characteristics, which has led many investigators to focus on cw ultrasound for acousto-optic image formation.

\subsection{The problem with continuous wave AOI}

There are basically two problems with using cw ultrasound in this context. First of all, cw sound beams afford only limited axial resolution, for the modulated photons emanate from a volume contained within the entire length of the beam. Spatial focusing can help, but for the most part $\mathrm{cw}$ is useful for 
generating image data possessing only transverse resolution. (Techniques such as frequency chirping [8], stroboscopic detection [9], and tomographic imaging [10] can yield limited axial resolution at the expense of systemic complexity).

The potential for deleterious bioeffects is another key issue with using $\mathrm{cw}$ ultrasound. The FDA has strict standards for ultrasound exposure that limits both the temporal-peak intensity and the temporal-average intensity of sound that one can safely transmit into the body. The former relates to mechanical effects such as cavitation, and establishes a limit on the peak intensity one can employ, regardless of whether one is working with $\mathrm{cw}$ or pulsed systems. The latter relates to thermal effects resulting from US absorption by tissue. These effects are greatly exacerbated when working at $\mathrm{cw}$ or with very long pulses, for the rate of sound power absorbed - and subsequently converted to heat - scales roughly with the duty cycle (the fraction of time the ultrasound is turned on). This is one reason why commercial diagnostic US imagers work at very low duty cycles (order $0.1 \%$ ). It is also one reason why high-intensity focused ultrasound therapy systems employ CW ultrasound to effect tissue ablation and thermal lesion formation.

\subsection{The case for pulsed AOI}

The use of short-pulse ultrasound to pump the AO signal offers several advantages. First of all, the signal reflects US spatial resolution in both the transverse (order 1-2 wavelengths) and the axial (order 2-3 wavelengths) directions. Secondly, one can work at much greater peak intensities, for tissue heating is not a significant factor. Higher peak acoustic intensity effects greater optical modulation depth and makes it easier to detect AO signals at greater imaging depths. Thirdly, this approach makes acousto-optic sensing automatically compatible with the large number of US imaging systems currently on the market. By using an ultrasound scanner to both generate US images and pump the AO signal, one can achieve true multi-mode acoustical and acousto-optic imaging such that image registration is automatic, instrumentation requirements are minimized, and FDA safety limits on ultrasound exposure are adhered to a priori.

Thus is the objective of the work described herein. We have developed an optical sensing system employing a photorefractive crystal (PRC) interferometer sensitive enough to work with conventional "B-Mode" pulsed ultrasound [11,12]. This technology is employed in conjunction with a commercial clinical scanner to generate dual-mode images of optically absorbing inclusions embedded in diffusive tissue phantoms and ex vivo biological tissue.

\section{EXPERIMENTAL ARRANGEMENT}

The enabling technology in this research effort is a PRC-based interferometry system for demodulating the AO signal generated in an optically diffusive medium using a commercial diagnostic ultrasound scanner. The operation of the PRC based interferometer has been detailed in the literature [11]. The experimental setup is given in Fig. 1. The output of a $230 \mathrm{~mW}$ frequency-doubled Nd:YAG laser is split into a signal beam and reference beam. The reference beam is directed around the test tank to a BSO crystal with dimensions $5 \times 5 \times 7 \mathrm{~mm}^{3}(\mathrm{X}, \mathrm{Z}$, $\mathrm{Y})$. The signal beam is sent through a $10 \mathrm{x}$ beam expander to the sample surface through the transparent walls of the test tank. The scattered light is collected and mixed with the reference in the photorefractive crystal. The signal and reference beams interfere in the PRC, creating a spatially varying intensity

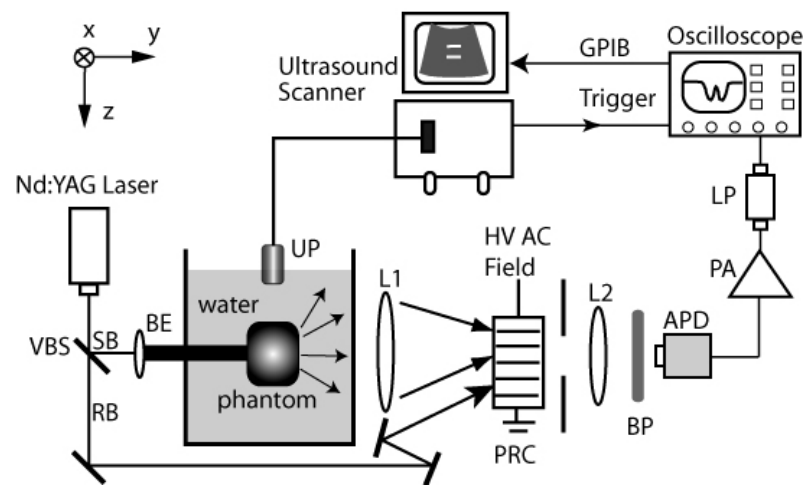

Figure 1. Experimental setup: VBS- variable beamsplitter, RB- reference beam, SB- signal beam, BE- beam expander, UP- ultrasound probe, L1, 2- lenses, PRC- photorefractive crystal, BP- optical bandpass filter, APD- avalanche photodiode, PA- preamplifier, LP- lowpass filter

pattern (grating) within the crystal. The generation and subsequent motion of free carriers within the crystal leads to the creation of a space-charge field, and finally the formation of an index of refraction grating through the electro-optic effect. In short, the spatially dependent intensity grating is written into the crystal as an index of refraction grating or hologram. A $10 \mathrm{kV} / \mathrm{cm}$ high voltage AC field is applied to the crystal to operate in the drift regime and enhance the two-wave mixing process. The reference beam is diffracted by the PRC hologram and interferes with the signal beam at an avalanche photodiode (APD) with a $10 \mathrm{~mm}$ sensing aperture. Note that the diffracted reference beam is an exact replica of the signal beam, less the phase modulation induced by the high frequency ultrasound source [11]. In our setup, the diffracted reference beam is inphase with the transmitted signal beam and the two interfere constructively at the detector. We therefore detect a net decrease in the optical intensity at the detector in the presence of the ultrasound source, irrespective of the sign of the phase modulation. This allows for coherent summation of the signals detected over multiple speckles, even though the signals are not spatially coherent at the ultrasound modulation frequency. In fact, the signal that is utilized for sensing and imaging applications is not at the ultrasound modulation frequency, but is rather a DC offset signal that is proportional to the instantaneous flux of modulated photons incident on the aperture of the sensor.

Returning to the experimental setup, the PRC-based optical detection system is combined with a commercially available, PC-based, diagnostic ultrasound scanner (AN2300, 


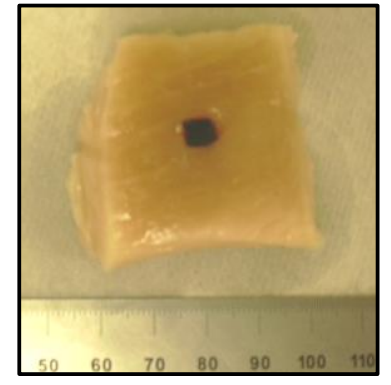

Figure 2. Cut-away view of the chicken breast sample used in the experiments. The ruler indicates the sample size in $\mathrm{mm}$, and the optical axis is perpendicular to the page $(2 \mathrm{~cm}$ thickness).

Analogic, Peabody, MA, USA). A commercially available ultrasound probe (5 MHz center frequency, 192-element, linear array, (B\&K Medical, Herlev, Denmark)) is excited by the AN2300 and projects ultrasonic pulses along the Z-axis to generate both ultrasound and $\mathrm{AO}$ images in the ZX-plane. A sample consisting of a $4.5 \times 2 \times 4.5 \mathrm{~cm}^{3}$ (X, Y and Z) slab of chicken breast was mounted between two parallel, transparent plexiglass plates of 1.3-mm thickness, oriented perpendicular to the optical axis. Prior to mounting, a cut was made midway through the breast across the $\mathrm{X}-\mathrm{Z}$ plane into which was inserted a $4 \mathrm{~mm}$ cube of poly-acrylamide gel doped with India ink (absorption coefficient $3 \mathrm{~cm}^{-1}$ ). A cut-away view of the chicken breast sample with the embedded inclusion is given in Fig. 2. With a sound speed of $1.5 \mathrm{~mm} / \mu \mathrm{s}$ and a density of 1.05 $\mathrm{mg} / \mathrm{mm}^{3}$, this target exhibited minimal acoustical contrast and significant optical contrast. To generate the US images, shortduration $5 \mathrm{MHz}$ Gaussian pulses (about 1.5-cycles long) were used. The ultrasound scanner demodulates the received ultrasound echo train associated with a given scan line and converts the signal envelope function to grayscale. In this way, a single ultrasonic shot can be used to build one "A line" and the full 2-D B-mode image is constructed from 192 A lines.

The envelope of an AO signal in the time-domain gives a measure of the intensity of the virtual light source generated along a given ultrasound scan line. However, the low SNR associated with AO sensing mandates that slightly longer duration pulses and time-averaging be used. The AO scans employ either 3- or 9-cycle long, $5 \mathrm{MHz}$ sine bursts. The spatial peak, temporal peak (SPTP) excursion of the sine bursts was typically $1 \mathrm{MPa}$ peak-negative and $6 \mathrm{MPa}$ peak-positive (measured in water). The pulse repetition frequency was about $1 \mathrm{kHz}$ and each AO signal was coherently averaged 40,000 times when using for 3-cycle pulses, and 10,000 times for 9cycle pulses. AO images were built in the same manner as the B-mode US images, and superimposed on top of the B-mode images using a color-scale. As a consequence, B-mode and AO images were automatically co-registered.

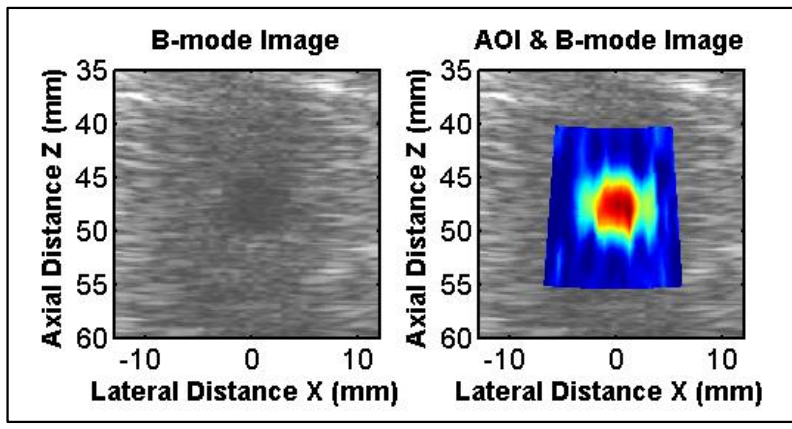

Figure 3. B-mode ultrasound image of inclusion (left) and Bmode image color-coded with the AOI information (right).

\section{EXPERIMENTAL RESULTS}

A typical $B$-mode ultrasound image of the sample, taken in the $\mathrm{XZ}$ plane through the center of the absorbing target, is shown in Fig. 3(left). The target appears as a dark region in the center of the image due to the reduced acoustic scattering from this region. Additional structure in the image can be attributed to acoustic backscatter from tissue microstructure. Figure 3(right) shows the corresponding AO image acquired using 3-cycle ultrasonic pulses. The AO image has been superimposed on the ultrasound image for comparison. The AO signals have been processed to remove the signal component from the background light distribution. In this processing, the signal observed along each scan line is fit to a Gaussian. This Gaussian fit, approximating the background light distribution, is then subtracted from the AO signal. This process is repeated along each scan line. The full width at half maximum of the imaged target along both the $\mathrm{X}$ and $\mathrm{Z}$ directions is approximately 5 $\mathrm{mm}$. A rough estimate of the expected target size, obtained through a convolution of the target shape with the spatial extent of the ultrasound pulse along the corresponding direction, agrees well with that observed experimentally.

Three-dimensional US and AO information can be obtained by mechanically scanning the ultrasound transducer in one dimension. The results of one such scan on the chicken breast sample are shown in Fig. 4. AO images were obtained in the $\mathrm{XZ}$ plane at 7 locations along the $\mathrm{Y}$-axis, each separated by $2 \mathrm{~mm}$. The target resides at approximately $\mathrm{Y}=0$. For this experiment, a 9-cycle ultrasound pulse was used to pump the AO interaction. A longer pulse allows for improved signal-tonoise ratio, or faster acquisition time, because of the increased AO interaction volume. This improvement comes, however, at the expense of axial resolution. The target is clearly resolved in all three dimensions. Line scans taken along the $\mathrm{Y}$ direction indicate that there is some broadening of the signal along the optical axis. This may be due to changes in the photon distribution near the target surface, or a mild shadowing effect. 


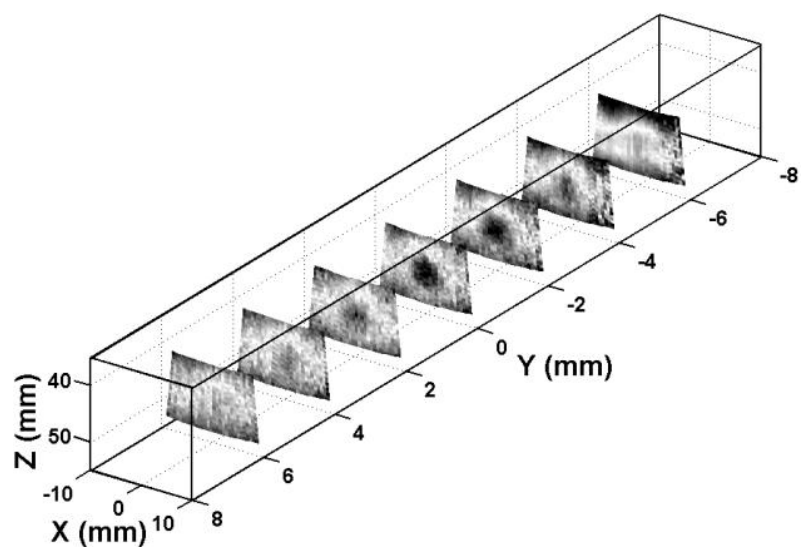

Figure 4. 2-D acousto-optic images acquired at several $\mathrm{XZ}$ planes along the $\mathrm{Y}$ axis. The absorber is centered at $\mathrm{Y}=0$ and clearly resolved along the $\mathrm{Y}$ - axis.

\section{DISCUSSION AND CONCLUSIONS}

The fact that our AO system is sufficiently sensitive for AOI using pulsed ultrasound is not only significant in improving the axial resolution of AOI, but also allows for the seamless marriage of AO and US imaging. We have developed a fused US/AO imaging system, and have used this to detect optical inhomogeneities in excised biological tissue. It is important to note that the results presented here were taken at a wavelength of $532 \mathrm{~nm}$, and improvements in signal-to-noise ratio and acquisition time are anticipated by moving to the NIR wavelength regime. We are currently in the process of developing a US/AOI imaging system operating at a substantially higher fluence rate (but below the MPE) at a wavelength of $1064 \mathrm{~nm}$.

A second technical hurdle that must be overcome before in-vivo measurements are possible concerns the response time of the detection system. The PRC continually rewrites the index grating with a characteristic response time that depends on both the crystal properties and incident light intensity. The response time must be fast enough to compensate for ambient or physiological motion, such that the diffracted reference beam remains matched to the signal beam. The response time of the BSO crystal used in these experiments, under our experimental conditions, was measured to be $150 \mathrm{~ms}$. This slow response time necessitated some degree of vibration isolation against ambient noise. This is clearly not suitable for in-vivo measurements where the speckle decorrelation time has been measured to be on the order of $1 \mathrm{~ms}$ [13]. In moving to the NIR wavelength range, however, semiconductor photorefractive crystals such as GaAs, InP, and $\mathrm{CdTeV}$ are available which, under some operating conditions, can reach response times in the desired range [14].

We argued previously that a principal advantage of pumping the AO response with pulsed ultrasound is that one can employ significantly higher peak intensities without incurring thermal bioeffects. However, there remains a risk of mechanical bioeffets associated with acoustic cavitation activity - a risk that grows greater with increasing pressure amplitude and decreasing acoustic frequency [15]. To assess the likelihood of generating cavitation, the FDA employs a parameter called the Mechanical Index (M.I.), defined as [16]:

$$
M . I . \equiv \frac{p_{s p t p}}{\sqrt{f}},
$$

where $p_{s p t p}$ is the derated spatial-peak temporal-peak negative pressure in MPa and $f$ is the US center frequency in MHz. The maximum permissible M.I. employed by diagnostic ultrasound machines sold in the U.S. is 1.8; anything greater is deemed too risky for clinical use. This places an upper bound on the acoustic pressures that one can expect to use to pump the AO response in vivo. Note that the M.I. used to acquire the images in Figs. 3 and 4 was only 0.45, which is well within the FDA limit.

Equation 1 suggests that $\mathrm{AOI}$ at higher frequencies is desirable for (i) it allows one to work at greater acoustic intensities and (ii) it affords improved spatial resolution. One can counter by pointing out that thermal effects are exacerbated at elevated frequencies, but that will only be an issue if using cw ultrasound; we have yet another argument for working with pulses. As a case in point, if one increases the US frequency from $1 \mathrm{MHz}$ to $8 \mathrm{MHz}$, the allowable peak negative pressure increases by a factor of 2.8 , and the resolution improves by a factor of 8. It is true that US penetration will also be reduced (due to greater attenuation), but at these frequencies the imaging depth will still be limited by optical rather than acoustical considerations. Future manifestations of our dual mode imaging system will therefore employ scan heads operating at higher center frequencies (order $10 \mathrm{MHz}$ ).

\section{ACKNOWLEDGEMENT}

The authors thankfully acknowledge the financial support of Center for Subsurface and Imaging Systems via NSF ERC award number EEC-9986821, and the assistance of Dr. Emmanuel Bossy in setting up the ultrasound instrumentation.

\section{REFERENCES}

[1] R. Muthupillai, D.J. Lomas, P.J. Rossman, J.F. Greenleaf, A. Manduca, R.L. Ehman, "Magnetic resonance elastography by direct visualization of propagating acoustic strain waves" Science 269, 1854, 1995.

[2] A.R. Skovoroda, S.Y. Emelianov, and M. O'Donnell, "Tissue elasticity reconstruction based on ultrasonic displacement and strain imaging," IEEE Trans UFFC 42, 747, 1995.

[3] A.P. Sarvazyan, Method and device for shear wave elasticity imaging. US Patent Number 5,606,971. 1997.

[4] J.G. Webster, Electrical Impedance Tomography, Adam Hilger, New York, 1990.

[5] V.V. Tuchin. Handbook of Optical Biomedical Diagnostics, SPIE Press, Washington, 2002. 
[6] D. Dolfi and F. Micheron, "Imaging process and system for transillumination with photon frequency marking," International Patent WO 89/00278, 1989.

[7] L.H.V. Wang, "Ultrasound-mediated biophotonic imaging: A review of acousto-optical tomography and photo-acoustic tomography," Disease Markers 19, 123, 2003.

[8] L.-H. Wang and G. Ku, "Frequency-swept ultrasound modulated optical tomography of scattering media," Opt. Lett. 23, 975, 1998.

[9] M. Atlan, B.C. Forget, F. Ramaz, A.C. Boccara, and M. Gross, "Pulsed acousto-optic imaging in dynamic scattering media with heterodyne parallel speckle detection", Opt. Lett. 30, 1360, 2005.

[10] J. Li and L.V. Wang, "Ultrasound modulated computed tomography of biological tissues," Appl. Phys. Lett. 84(9), 1597 (2005)

[11] T.W. Murray, L. Sui, G. Maguluri, R.A. Roy, A. Nieva, F. Blonigen and C.A. DiMarzio, "Detection of Ultrasound Modulated Photons in Diffuse Media Using the Photorefractive Effect”, Opt. Lett. 29, 2509, 2004.

[12] E. Bossy, L. Sui, T.W. Murray, and R.A. Roy, "Fusion of conventional ultrasound imaging and acousto-optical imaging using a standard pulsed ultrasound scanner", Opt. Lett. 30, 744, 2005.

[13] M. Gross, P. Goy, B.C. Forget, M. Atlan, F. Ramaz, A.C. Boccara, and A.K. Dunn, "Heterodyne detection of multiply scattered monochromatic light with a multipixel detector," Opt. Lett. 30, 1357, 2005.

[14] J. Millerd, M. Ziari, and A. Partovi, "Photorefractivity in semiconductors," in Semiconductors and Semimetals Vol. 58, Academic Press, New York, 1999.

[15] R.E. Apfel and C.K. Holland. Gauging the Likelihood of Cavitation from Short-Pulse, Low-Duty Cycle Diagnostic Ultrasound. Ultrasound in Med. and Biol. 17,179-185, 1991.

[16] "Information for manufacturers seeking marketing clearance of diagnostic ultrasound systems and transducers," U.S. Department of Health and Human Services, Food and Drug Administration, Center for Devices and Radiological Health, 1997. 